\title{
ON $S^{3}$-EQUIVARIANT HOMOLOGY
}

\author{
MOHAMED ELHAMDADI
}

(Received 12 September 2000)

\begin{abstract}
We prove that the group $S^{3}$ (norm 1 quaternions) cannot be a geometric realization of a crossed simplicial group and construct an exact sequence connecting $S^{3}$ equivariant homology of an $S^{3}$-space with its Pin(2)-equivariant homology.
\end{abstract}

2000 Mathematics Subject Classification. 19G38, 55T05, 22E40.

1. Introduction. This paper arose from a desire to better understand the topological interpretation of quaternionic homology given in [4]. Because of the four-fold periodicity of this homology, one wants the existence of a small category $\mathscr{b}$ such that its classifying space $B \mathscr{C}$ is equal to the classifying space of the Lie group $S^{3}$. This is the analogue of the result concerning the category $\Lambda$ such that its classifying space is homotopically equivalent to the classifying space of the circle $S^{1}$ (see [1]). The first result in the former case was obtained by Dwyer et al. [2] giving the $p$-completion of $B S^{3}$ for any prime number $p$. For $p=2$, they give an explicit way of constructing the 2-completion of $B S^{3}$ using some finite subgroups of $S^{3}$. The $p$-completion, for $p$ odd prime, of infinite quaternionic projective space $B S^{3}$ is the same as the $p$-completion of the classifying space of the normalizer Pin(2) of a maximal torus in $S^{3}$. Fiedorowicz and Loday [3] generalized Connes' notion of the cyclic category $\Lambda$ by introducing the category of crossed simplicial groups with simplicial groups as objects and crossed group homomorphism as morphisms (see Definition 3.1). The geometric realization of a crossed simplicial group $G_{*}$ is a topological group $\left|G_{*}\right|$. Theorem 5.15 of [3] restricts the kinds of topological groups, including the Lie group $S^{3}$, which can result from geometric realization. In Proposition 3.11 of [4], Loday defined a category $\Delta Q$ such that its classifying space is homeomorphic to $B \operatorname{Pin}(2)$ and showed that quaternionic homology is Pin(2)-equivariant homology. Combining this result and a long exact sequence connecting the $S^{3}$-equivariant homology of an $S^{3}$-space with its $\operatorname{Pin}(2)$-equivariant homology (Theorem 4.1), we deduce that if 2 is invertible in the ground field $k, A$ is a $k$-algebra with involution and $Y$ is the geometric realization of the quaternionic simplicial $k$-module associated to $A$ (see [3] for complete definition), then quaternionic homology becomes an $S^{3}$-equivariant homology and the Connes's exact sequence for quaternionic homology becomes the Gysin exact sequence of an $S^{3}$-fibration. We are currently working on linking the two concepts when 2 is not invertible in the ground field.

2. Preliminaries on quaternionic homology. Let $A$ be an involutive unital $k$-algebra where $k$ is a commutative ring. When the set of rationals $\mathbb{Q}$ is contained in $k$, 
quaternionic, respectively dihedral homology (here they coincide because 2 is invertible in $k$ ), can be defined as the homology of the coinvariant space of $A^{\otimes(n+1)}$ for the action of the quaternionic group $Q_{n+1}=\left\langle x, y \mid x^{n+1}=y^{2}, y x y^{-1}=x^{-1}\right\rangle$ (respectively, the dihedral group $\left.D_{n+1}=\left\langle x, y \mid x^{n+1}=y^{2}=1, y x y^{-1}=x^{-1}\right\rangle\right)$ usually denoted by $\left(A^{\otimes(n+1)} /(1-x, 1-y)\right) . H Q_{n}(A)=H_{n}\left(A^{\otimes(*+1)} /(1-x, 1-y), b\right)$, where $b$ is the Hochschild boundary $b=\sum_{i=0}^{n-1}(-1)^{i} d_{i}$, and the generators $x$ and $y$ act on $A^{\otimes(n+1)}$ by $x\left(a_{0}, a_{1}, \ldots, a_{n}\right)=(-1)^{n}\left(a_{n}, a_{0}, \ldots, a_{n-1}\right)$ and $y\left(a_{0}, a_{1}, \ldots, a_{n}\right)=(-1)^{n(n+1) / 2}\left(\bar{a}_{0}, \bar{a}_{n}\right.$, $\left.\bar{a}_{n-1}, \ldots, \bar{a}_{1}\right)$. For the case when 2 is not invertible, Loday [4] defined the quaternionic homology as the homology of the total complex of a bicomplex obtained using a free periodic resolution of period four of $\mathbb{Z}$ as trivial $Q_{n+1}$-module and gives an important result on quaternionic homology which is the periodicity exact sequence

$$
\cdots \rightarrow H T_{n}(A) \longrightarrow H Q_{n}(A) \longrightarrow H Q_{n-4}(A) \longrightarrow H T_{n-1}(A) \longrightarrow \cdots,
$$

where $H T_{*}$ is the homology of a complex $T_{*}$ obtained by elimination of acyclic complex in the bicomplex (see [4] for more details). The theory $H T$ is to quaternionic homology as Hochschild homology is to cyclic homology.

3. Crossed simplicial groups. Using the notion of crossed simplicial groups (Definition 3.1) and their homology [3], the quaternionic homology can be understood as Pin(2)-equivariant homology instead of $S^{3}$-equivariant homology which seems to be the natural candidate because of the fourth periodicity. The reason why it is not the latter homology is connected to the next Lemma 3.2. We will then in the main theorem connect these two homologies.

DEFINITION 3.1 (see [3]). A crossed simplicial group is a family of groups $\left\{G_{n}\right\}_{n \geq 0}$ such that there exists a small category $\Delta G$ with the following properties:

(1) The objects of $\Delta G$ are ordered sets $[n]=\{0,1, \ldots, n\}$.

(2) $\Delta G$ contains the simplicial category $\Delta$ as a subcategory.

(3) The automorphism group of [ $n]$ in $\Delta G$ is the group $G_{n}^{\text {op }}$ (opposite group of $G_{n}$ ).

(4) Any morphism from [ $n]$ to $[m]$ in $\Delta G$ can be uniquely written as a composite $\Phi \circ g$, where $\Phi \in \operatorname{Hom}_{\Delta}([n],[m])$ and $g \in \operatorname{Aut}_{\Delta G}([n])=G_{n}^{\text {op }}$.

The classical examples (see [3]) are the family of cyclic groups $\{\mathbb{Z} / m \mathbb{Z}\}_{m \geq 1}$, dihedral groups $\left\{D_{m}\right\}_{m \geq 1}$, quaternionic groups $\left\{Q_{m}\right\}_{m \geq 1}$, and the family of permutation groups $\left\{S_{m}\right\}_{m \geq 1}$. The geometric realizations of these crossed simplicial groups are, respectively, the circle group $S^{1}$, the orthogonal group $O(2)$, the normalizer of $S^{1}$ in $S^{3}$, and the infinite sphere $S^{\infty}=\lim _{n} S^{n}$. Then a natural question arises: does there exist a crossed simplicial group such that its geometric realization is the Lie group $S^{3}$ ?

\subsection{The Lie group $S^{3}$ is not a crossed simplicial group}

LEMMA 3.2. The group $S^{3}$ is not nilpotent.

Proof. This is because, if $S^{3}$ were nilpotent, there would exist $q$ normal subgroups $H_{1}, H_{2}, \ldots, H_{q}$ of $S^{3}$ such that $S^{3}=H_{0} \supset H_{1} \supset \cdots \supset H_{q}=\{1\}$ and for all $k$, $0 \leq k \leq q-1$, there would be an inclusion $H_{k} / H_{k+1} \subset \operatorname{center}\left(S^{3} / H_{k+1}\right)$. In particular, $H_{q-1} \subset \operatorname{center}\left(S^{3}\right)=\{ \pm 1\}$. We can assume that the inclusion $H_{q} \subset H_{q-1}$ is strict, which 
implies $H_{q-1}=\{ \pm 1\}$. In the same way, $H_{q-2} / H_{q-1} \subset \operatorname{center}\left(S^{3} /\{ \pm 1\}\right)=\{1\}$ because $S^{3} /\{ \pm 1\}$ is simple. This implies that $H_{q-2}=H_{q-1}$. So the sequence of inclusions reduces to $S^{3}=H_{0} \supset H_{1}=\{ \pm 1\} \supset H_{2}=\{1\}$ and then $O^{+}(3, \mathbb{R})=S^{3} /\{ \pm 1\}=H_{0} / H_{1} \subset$ $\operatorname{center}\left(S^{3} / H_{1}\right)=\{1\}$, giving us a contradiction.

THEOREM 3.3 (see [3]). If $G_{*}$ is a crossed simplicial group such that the geometric realization $\left|G_{*}\right|$ is a Lie group, then the path component of the identity of $\left|G_{*}\right|$ is nilpotent.

As a consequence of Lemma 3.2 and Theorem 3.3, there is no crossed simplicial group with geometric realization $S^{3}$.

Another approach to the question is to consider the discrete subgroups of $S^{3}$. For this we need to recall the following theorem.

THEOREM 3.4 (see [5]). Every finite subgroup of $S^{3}$ is a cyclic, binary dihedral, or binary polyhedral group. If two finite subgroups of $S^{3}$ are isomorphic, then they are

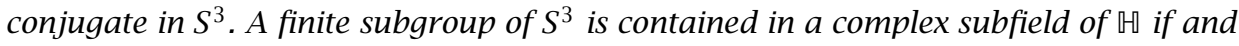
only if it is cyclic, and is contained in the real subfield of $\mathbb{W}$ if and only if it is cyclic of order 1 or 2.

Based on this theorem, we see that $S^{3}$ cannot be a crossed simplicial group. In fact, if the topological group $S^{3}$ were a crossed simplicial group $S^{3}=\left|G_{*}\right|$, then by Proposition 5.13 in [3], there would be inclusions of discrete subgroups $G_{n} \subset S^{3}$. Moreover, the discrete subgroups of $S^{3}$ are, up to conjugations, the families of cyclic subgroups $\{\mathbb{Z} / n \mathbb{Z}\}$, those of quaternionic $\left\{Q_{n}\right\}$, the binary tetrahedral group, the binary octahedral, and the binary icosahedral. In addition, the geometric realizations of these five simplicial groups give, respectively, the circle $S^{1}$, the group Pin(2), the binary tetrahedral group, the binary octahedral group, and the binary icosahedral group and therefore they cannot give $S^{3}$.

4. $S^{3}$-equivariant homology. Let $G$ be a group and $\mathscr{E} G$ be the category with one object $*$ such that the monoid $\operatorname{Hom}_{\mathscr{E} G}(*, *)$ is the group $G$. The geometric realization of the nerve of this category is a contractible space denoted by $E G=|\mathscr{E} G|$. The group $G$ acts transitively on $E G$ and the orbit space is the classifying space $B G$ of the group $G$. In fact there is a principal $G$-bundle $E G \rightarrow B G$.

For a $G$-space $Y$, the Borel space is the quotient of $E G \times Y$ by the equivalence relation generated by $(g x, g z) \sim(x, z)$ for all $g \in G$ and $x, z \in Y$. This space is usually denoted by $E G \times{ }_{G} Y$. Recall that there is a fibration

$$
G \rightarrow E G \times Y \rightarrow E G \times{ }_{G} Y
$$

The $G$-equivariant homology of $Y$ is, by definition, the homology of the associated Borel space $H_{n}^{G}(Y, k):=H_{n}\left(E G \times_{G} Y, k\right)$.

The main result of this paper is the following theorem.

THEOREM 4.1. Let $Y$ be a connected $S^{3}$-space. There is a long exact sequence

$$
\cdots \rightarrow H_{n-1}^{S^{3}}(Y, \mathbb{Z} / 2 \mathbb{Z}) \longrightarrow H_{n}^{\operatorname{Pin}(2)}(Y) \rightarrow H_{n}^{S^{3}}(Y) \rightarrow H_{n-2}^{S^{3}}(Y, \mathbb{Z} / 2 \mathbb{Z}) \longrightarrow \cdots
$$


Proof. The inclusion $\operatorname{Pin}(2) \rightarrow S^{3}$ induces the following fibration:

$$
\mathbb{R} \mathbb{P}(2) \longrightarrow E \operatorname{Pin}(2) \times_{\operatorname{Pin}(2)} Y \longrightarrow E S^{3} \times_{S^{3}} Y .
$$

Since $H_{q}(\mathbb{R} \mathbb{P}(2))=0$ for $q \neq 0$ and $q \neq 1$, the spectral sequence lies on the two horizontal lines $q=0$ and $q=1$. The only nonzero differential is $d^{2}$. The filtration of $H_{n}^{\text {Pin(2) }}(Y)$ is given by

$$
0=F_{0} \subset F_{1} \subset \cdots \subset F_{n-1} \subset F_{n} H_{n}^{\mathrm{Pin}(2)}(Y)=H_{n}^{\mathrm{Pin}(2)}(Y) .
$$

The successive quotients are given by

$$
\frac{H_{n}^{\operatorname{Pin}(2)}(Y)}{F_{n-1}}=E_{n, 0}^{\infty}=\operatorname{ker}\left(H_{n}^{S^{3}}(Y) \rightarrow H_{n-2}\left(E S^{3} \times_{S^{3}} Y ; \mathbb{Z} / 2 \mathbb{Z}\right)\right),
$$

$F_{n-1} / F_{n-2}=E_{n-1,1}^{\infty}$, and $F_{n-2}=F_{n-3}=\cdots=F_{0}=0$ because $E_{p, q}^{\infty}=0$ since $q \geq 2$.

Thus we have the exact sequence,

$$
0 \longrightarrow E_{n-1,1}^{\infty} \longrightarrow H_{n}^{\mathrm{Pin}(2)}(Y) \longrightarrow E_{n, 0}^{\infty} \longrightarrow 0 .
$$

We also have

$$
E_{n, 1}^{\infty}=E_{n, 1}^{3}=\frac{H_{n}\left(E S^{3} \times_{S^{3}} Y ; \mathbb{Z} / 2 \mathbb{Z}\right)}{\operatorname{Im}\left(H_{n+2}^{S^{3}}(Y) \longrightarrow H_{n}\left(E S^{3} \times_{S^{3}} Y ; \mathbb{Z} / 2 \mathbb{Z}\right)\right)} .
$$

Therefore, the filtration of $H_{n}^{\text {Pin(2) }}(Y)$ becomes

$$
0 \subset F_{n-1}=\frac{H_{n-1}\left(E S^{3} \times_{S^{3}} Y ; \mathbb{Z} / 2 \mathbb{Z}\right)}{\operatorname{Im}\left(H_{n+1}^{S^{3}}(Y) \longrightarrow H_{n-1}\left(E S^{3} \times_{S^{3}} Y ; \mathbb{Z} / 2 \mathbb{Z}\right)\right)} \subset F_{n}=H_{n}^{\mathrm{Pin}(2)}(Y),
$$

and the quotient becomes

$$
\frac{F_{n}}{F_{n-1}}=\frac{H_{n}^{\operatorname{Pin}(2)}}{F_{n-1}} \cong \operatorname{ker}\left(H_{n}^{S^{3}}(Y) \longrightarrow H_{n-2}\left(E S^{3} \times_{S^{3}} Y ; \mathbb{Z} / 2 \mathbb{Z}\right)\right) .
$$

Then we obtain the exact sequence

$$
0 \longrightarrow E_{p, 0}^{\infty} \longrightarrow E_{p, 0}^{2} \stackrel{d^{2}}{\longrightarrow} E_{p-2,1}^{2} \longrightarrow E_{p-2,1}^{\infty} \longrightarrow 0 .
$$

Now, by combining (4.6) and (4.10), the exact sequence follows.

COROLLARY 4.2. If 2 is invertible in the field $k$, and $A$ is a $k$-algebra with involution, then the geometric realization of the quaternionic simplicial $k$-module associated to $A,\left\{A^{\otimes(n+1)}\right\}_{n \geq 0}$, allows one to obtain the periodicity exact sequence in quaternionic homology,

$$
\cdots \rightarrow H T_{n}(A) \longrightarrow H Q_{n}(A) \longrightarrow H Q_{n-4}(A) \longrightarrow H T_{n-1}(A) \longrightarrow \cdots,
$$

as the Gysin exact sequence of an $S^{3}$-fibration.

When 2 is invertible in $k$ and if $Y$ is the geometric realization of the simplicial module $\left\{A^{\otimes(n+1)}\right\}_{n \geq 0}$, then the mapping $E \operatorname{Pin}(2) \times_{\operatorname{Pin}(2)} Y \rightarrow E S^{3} \times S_{S^{3}} Y$ induces homology isomorphisms.

The groups $H_{*}^{S^{3}}(Y, \mathbb{Z} / 2 \mathbb{Z})$ look like obstruction to the isomorphisms $H_{*}^{\operatorname{Pin}(2)}(Y) \cong$ $H_{*}^{S^{3}}(Y)$.

ACKNOwLeDgement. I would like to thank Professor Hilton for his valuable discussions and insightful comments. 


\section{REFERENCES}

[1] A. Connes, Cohomologie cyclique et foncteurs $\mathrm{Ext}^{n}$ [Cyclic cohomology and functors $\mathrm{Ext}^{n}$ ], C. R. Acad. Sci. Paris Sér. I Math. 296 (1983), no. 23, 953-958 (French). MR 86d:18007. Zbl 534.18009.

[2] W. G. Dwyer, H. R. Miller, and C. W. Wilkerson, The homotopic uniqueness of BS ${ }^{3}$, Algebraic Topology, Barcelona, 1986, Lecture Notes in Math., vol. 1298, Springer, Berlin, 1987, pp. 90-105. MR 89e:55019. Zbl 654.55015.

[3] Z. Fiedorowicz and J.-L. Loday, Crossed simplicial groups and their associated homology, Trans. Amer. Math. Soc. 326 (1991), no. 1, 57-87. MR 91j:18018. Zbl 755.18005.

[4] J.-L. Loday, Homologies diédrale et quaternionique [Dihedral and quaternionic homologies], Adv. in Math. 66 (1987), no. 2, 119-148 (French). MR 89e:18024. Zbl 627.18006.

[5] J. A. Wolf, Spaces of Constant Curvature, McGraw-Hill, New York, 1967. MR 36\#829. Zbl 162.53304 .

MOHAmed ElHamdadi: Department of Mathematics, University of SOUTH Florida, 4202 EAST FOWLER AVENUE, PHY 114, TAMPA, FL 33620, USA

E-mail address: emohamed@math.usf.edu 


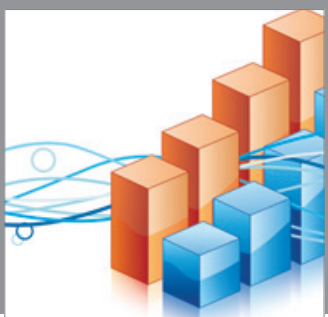

Advances in

Operations Research

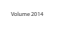

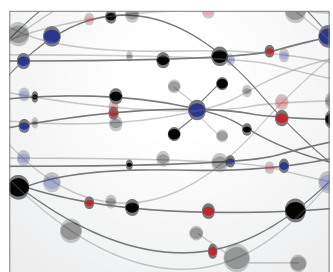

\section{The Scientific} World Journal
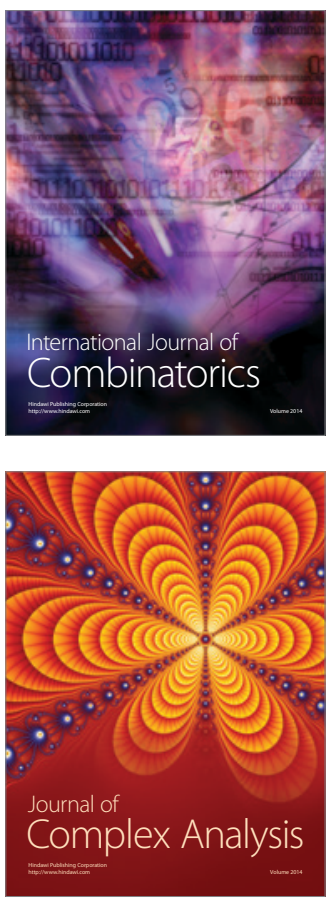

International Journal of

Mathematics and

Mathematical

Sciences
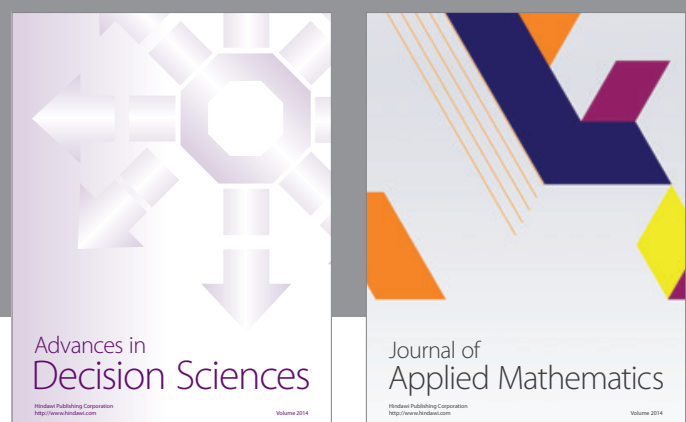

Journal of

Applied Mathematics
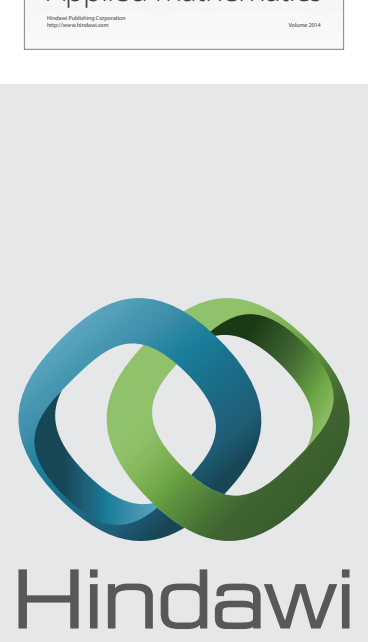

Submit your manuscripts at http://www.hindawi.com
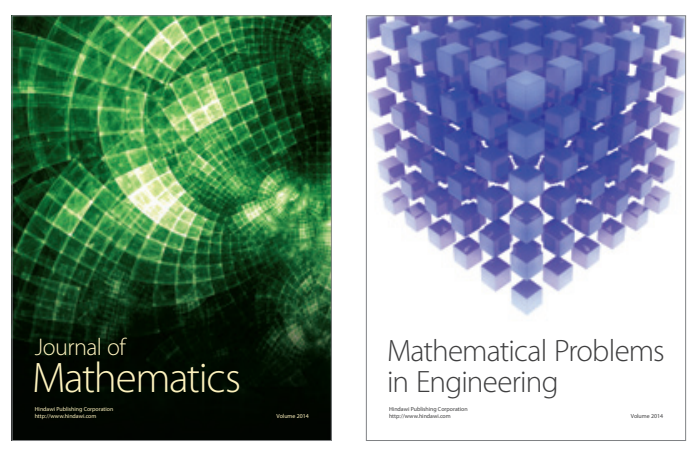

Mathematical Problems in Engineering
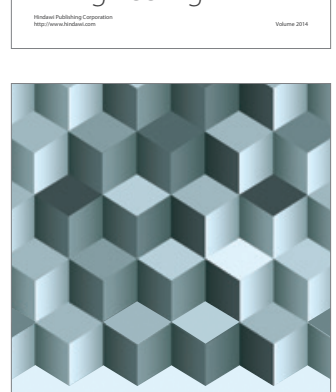

Journal of

Function Spaces
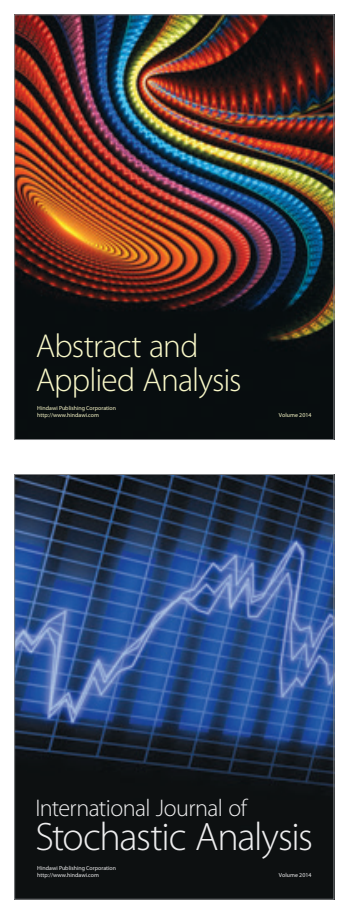

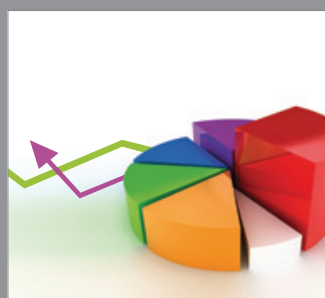

ournal of

Probability and Statistics

Promensencen
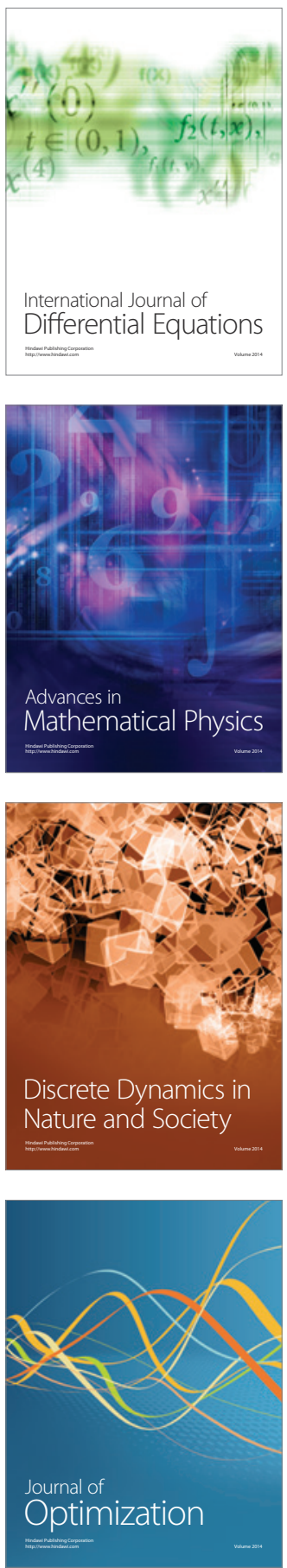